\title{
Antibiotics Induced Mitochondrial Toxicity: "a Neglected Factor Possibly Contributing to Tumorigenesis and Neurodegeneration"
}

\author{
R.L. Elliott ${ }^{1}$ and X.P. Jiang ${ }^{1}$ \\ ${ }^{1}$ Sallie Astor Burdine Breast Foundation, Baton Rouge, Louisiana 70806
}

In the 1920s Warburg reported that cancer cells have defective mitochondrial respiration resulting in a glycolytic metabolic pathway [1]. We demonstrated by normal mitochondrial organelle transplantation into cancer cells an inhibition of glycolytic metabolism [2]. The fact that mitochondria are evolutionary bacteria prompted us to study the deleterious effects of antibiotics on their relative, the mitochondria.

Antibiotic treatment can cause many side effects in patients including ototoxicity, nephrotoxicity, and disruption of the microbiome [3]. Antibiotics target and suppress bacteria and their components, erythromycins target large ribosomes while tetracyclines target the small ribosomes. Bacterial ribosomes are similar to mitochondrial ribosomes. Quinolones target bacterial gyrases and mitochondrial DNA topoisomerases. We investigated mitochondrial toxicity of antibiotics in human mammary epithelia and the potential risks of antibiotic usage impacting human diseases, such as cancer and neurodegeneration. Three antibiotics azithromycin (erythromycin) doxycycline (tetracycline) and ciprofloxacin (quinolone) were used to treat human mammary epithelial MCF-12A cell line.

Most cell lines for cell culture when received are cultured with an antibiotic. Therefore, we conducted our experiments in antibiotic free media. We compared effects on the MCF-12A cell line in gentamycin media to gentamycin free media. The MCF-12A human mammary epithelia normal cell line began to proliferate into a ductal pattern in the antibiotic free media (Fig. 1). This was dramatic demonstration of antibiotic toxic effect. Mitochondrial morphology was examined by electron and fluorescent microscopy. Mitochondrial membrane potential gradient and reactive oxygen species (ROS) were studied by fluorescent probe staining with fluorescent microscopy. Cell metabolism gene expression was measured by quantitative real time PCR (polymerase chain reaction). Antibiotics inhibited the proliferation of MCF-12A cells in a dose dependent pattern. Ultrastructure microscopy revealed that antibiotics cause vacuolated, swollen mitochondria with disrupted cristae (Fig. 2). MCF-12A cells were treated with IC50 concentration of antibiotics for 3 hours. The antibiotics significantly or completely suppressed mitochondrial membrane potential (JC-1 aggregates with bright red fluorescence). However, surviving cells recover from antibiotic inhibition and regain red JC-1 fluorescence at 48 hours and 7 days of antibiotic incubation (Fig. 3). All three antibiotics increased mitochondrial ROS production and real time PCR confirmed the microscopy findings. Three hours of antibiotic treatment caused decreased gene expression of mitochondrial oxidase phosphorylation (OXPHOS) enzymes, MT-ATP6 (mitochondrially encoded ATP synthase 6), MT-CYB (mitochondrially encoded cytochrome b), MT-CO1 (mitochondrially encoded cytochrome C oxidase 1) and MT-ND1 (mitochondrially encoded NADH dehydrogenase 1). After the initial stage of mitochondrial inhibition, MCF-12A cells gradually upregulate gene expression of HIF1-a (hypoxia inducible factor 1- alpha), MT-ATP6, MT-CYB, MTCO1, MT-ND1, and mitochondrial biogenesis transcription factors of PGC1a (peroxisome proliferator activated receptor gamma coactivator-1-alpha), NRF1 and NRF2 (nuclear respiratory factor 1 and 2) and Tfam (transcription factor A, mitochondrial). (Fig. 4) The expression of glycolysis related genes such as HK2 (hexokinase), PFKM (phosphofructokinase 1), PKM2 (pyruvate kinase) and LDHA (lactate dehydrogenase A) are also increased. Gene expression levels reach to peak levels at 7 days of antibiotic 
treatment. In response to ROS overproduction, MCF-12A cells upregulate gene expression of several antioxidants, catalase, superoxide dismutase (SOD) and glutathione peroxidase (GPX).

In conclusion, antibiotics induce mitochondrial dysfunction, ROS overproduction and increased glycolysis. Increased ROS can damage genomic and mitochondrial DNA especially in humans with mutated antioxidant genes [4-5]. Prolonged increase in HIF-1a and glycolysis might induce oncogenes and increased ROS, and oncogenes are associated with many diseases. Thus, overusage of antibiotics could contribute to tumorigenesis and neurodegeneration [6].

\section{References}

[1] Warburg O et al (1930), In: O. Warburg. Ed., The metabolism of tumors, Constable, Princeton, pp254-270

[2] Jiang XP et al (2015), Breast Cancer Research and Treatment 153:219-29.

[3] Elliott RL et al (2017), Antibiotic overusage causes mitochondrial dysfunction which may promote tumorigenesis 5(4):62-65.

[4] Ames BN et al (1993), Proc Natl Acad Sci USA 90(17):7915-22.

[5] Sullivan LB and Chandel NS (2014), Cancer and metabolism 2:17.

[6] Acknowledge funding from Sallie A. Burdine Breast Foundation, Baton Rouge, Louisiana.

Fig.1

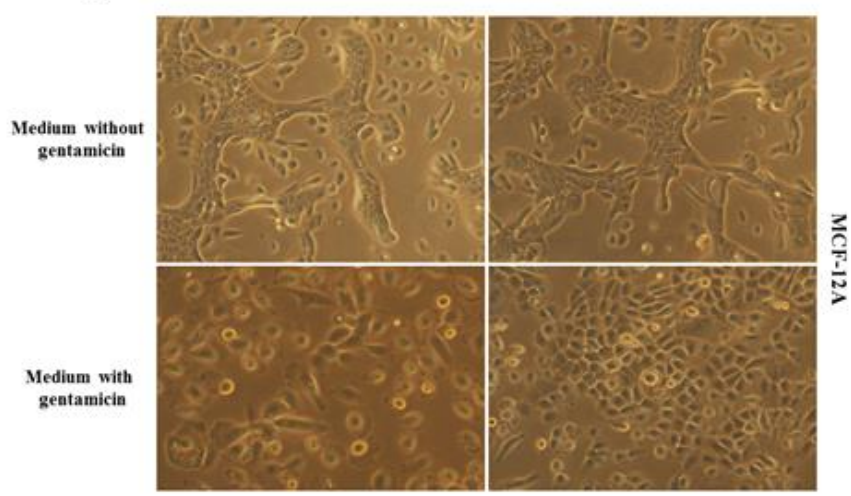

Fig. 3

MCF-12A cells recover mitochondrial membrane potential from the inhibition of azithromycin $(125 \mu \mathrm{M})$
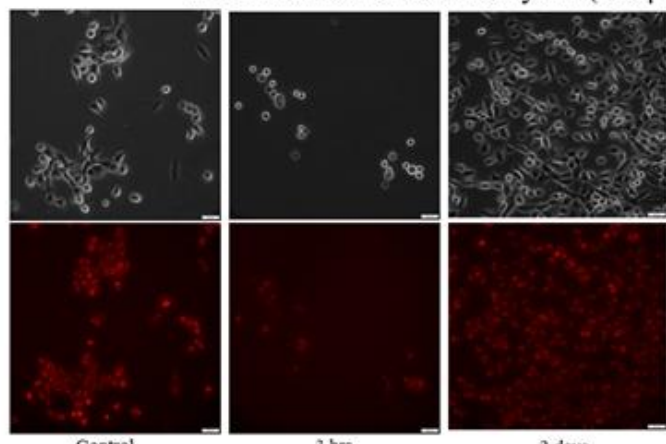

2 days

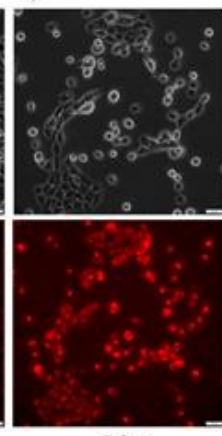

Fig. 2
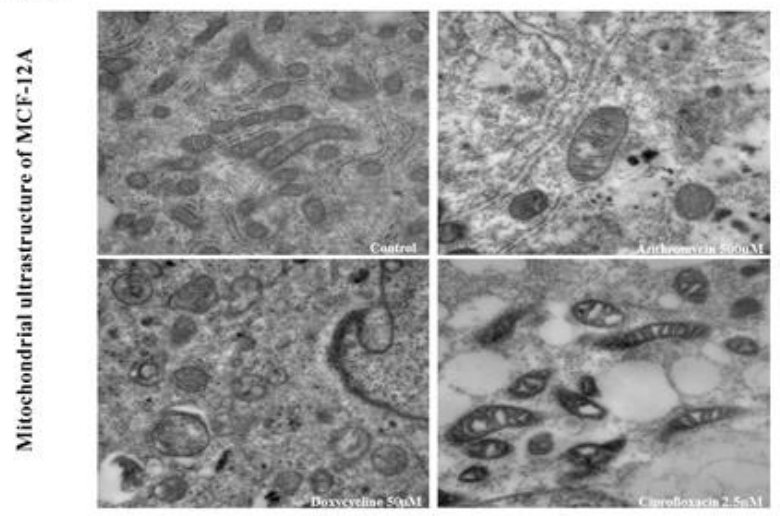

Fig. 4

Effects of azithromycin on gene expression of mitochondrialOXPHOS enzymes in MCF-12A cells.

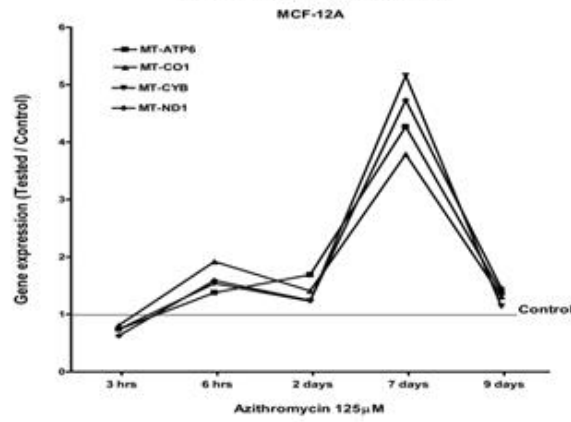

